\title{
NDD. 09. Antioxidant effects of melatonin administration in patients with cronic obstrutive pulmonary disease (COPD)
}

\author{
LOYOLA, L. ${ }^{a}$, PINHO, R. ${ }^{b}$, COUTINHO, G. ${ }^{c}$, BAIMA, C. ${ }^{c}$, BRUIN, P.F.C. ${ }^{d}$ \\ aUndergrad in Pharmacy, Universidade Federal do Ceará, Brazil \\ bPhD student, Medical Sciences, Universidade Federal do Ceará, Brazil \\ "Master degree student in Medical Sciences, Universidade Federal do \\ Ceará, Brazil \\ dMedical Sciences Department faculty, Universidade Federal do Ceará, \\ Brazil
}

Introduction: The Chronic Obstructive Pulmonary Disease (COPD) is a global health problem of growing importance and the current treatment available does not improve disease progression or its mortality (RODRIGUES, 2009). In this context, it is of utmost importance the search for new treatments for COPD. It is known that oxidative stress plays an important role in COPD, through direct damage to the components of the respiratory tract, as well as other mechanisms amplifying its pathophysiology. Objectives: This study aimed to clinically evaluate the use of the use of melatonin (MLT) for the treatment of COPD. MLT, the main product of pineal gland, is recognized as an important factor in the regulation of sleep, is also considered having antioxidant effects. Methods: This is an undergoing prospective clinical trial, double-blind, randomized, with three parallel groups without crossover. Sixty patients are enrolled and MLT-treated for 3 (three) months and distributed in three (3) groups, as follows: I. control, II. MLT 3mg, III. MLT 6mg. Each group receives one (1) tablet containing melatonin, and the group I receives one tablet of placebo, group II, one tablet of MLT (3mg) and Group III, one tablet of MLT (6mg). All tablets (MLT and placebo) are identical in all presentations of shape, size and color. All doses are administered one hour before the usual time of sleep. Patients are monitored by quizzes (Scale Epworth Sleepiness, Index of Sleep Quality of Pittsburgh (PSQI), Scale of Dyspnea of Medical Research Council (MRC), Saint George's Respiratory Questionnaire (SGRQ)) and their exhaled air assessed to evaluate the antioxidant effect. Conclusions: The medicines already used by patients before the study should be maintained. Results: To date, ten (10) patients received full treatment. They state improvements in quality of life, and especially, improvements in quality of sleep. Only when the sixty patients complete full treatment, one can disclose the placebo and the treatment groups (3mg and 6mg). Conclusions: Our first exploratory results suggest that the melatonin-treated patients have improved quality of life, however only when the study is concluded the full data will be disclosed.

LOYOLA, L.; PINHO, R.; COUTINHO, G.; BAIMA, C.; BRUIN, P.F.C. 2013. Antioxidant effects of melatonin administration in patients with cronic obstrutive pulmonary disease (COPD), p.38. In: Oriá, Reinaldo Barreto; Andrade, Geanne Matos de; Bruin, Veralice Meireles S. de. I International Symposium in Neuroscience Meeting [Blucher Neuroscience Proceedings n.1 v.1]. São Paulo: Blucher, 2014 http://dx.doi.org/10.5151/isnm-sine33 